\title{
ARTICLE
}

\author{
Received 5 Jul 2016 | Accepted 9 Dec 2016 | Published 1 Feb 2017
}

DOl: $10.1038 /$ ncomms14224

OPEN

\section{Self-surface charge exfoliation and electrostatically coordinated 2D hetero-layered hybrids}

Min-Quan Yang ${ }^{1}$, Yi-Jun $\mathrm{Xu}^{2,3}$, Wanheng Lu${ }^{4}$, Kaiyang Zeng ${ }^{4}$, Hai Zhu ${ }^{5}$, Qing-Hua Xu ${ }^{5} \&$ Ghim Wei Ho

At present, the technological groundwork of atomically thin two-dimensional (2D) hetero-layered structures realized by successive thin film epitaxial growth is in principle constrained by lattice matching prerequisite as well as low yield and expensive production. Here, we artificially coordinate ultrathin 2D hetero-layered metal chalcogenides via a highly scalable self-surface charge exfoliation and electrostatic coupling approach. Specifically, bulk metal chalcogenides are spontaneously exfoliated into ultrathin layers in a surfactant/intercalator-free medium, followed by unconstrained electrostatic coupling with a dissimilar transition metal dichalcogenide, $\mathrm{MoSe}_{2}$, into scalable hetero-layered hybrids. Accordingly, surface and interfacial-dominated photocatalysis reactivity is used as an ideal testbed to verify the reliability of diverse 2D ultrathin hetero-layered materials that reveal high visible-light photoreactivity, efficient charge transfer and intimate contact interface for stable cycling and storage purposes. Such a synthetic approach renders independent thickness and composition control anticipated to advance the development of 'design-and-build' 2D layered heterojunctions for large-scale exploration and applications.

\footnotetext{
${ }^{1}$ Department of Electrical and Computer Engineering, National University of Singapore, 4 Engineering Drive 3, 117583 Singapore, Singapore. ${ }^{2}$ State Key Laboratory of Photocatalysis on Energy and Environment, College of Chemistry, Fuzhou University, Fuzhou 350002, China. ${ }^{3}$ College of Chemistry, New Campus, Fuzhou University, Fuzhou 350108, China. ${ }^{4}$ Department of Mechanical Engineering, National University of Singapore, 9 Engineering Drive 1 , 117576 Singapore, Singapore. ${ }^{5}$ Department of Chemistry, National University of Singapore, 3 Science Drive 3, 117543 Singapore, Singapore. Correspondence and requests for materials should be addressed to G.W.H. (email: elehgw@nus.edu.sg).
} 
ltimate two-dimensional (2D) anisotropy with atomically thick layered structure is both an ideal low-dimensional system for fundamental study and an elemental building block for designed assembly ${ }^{1-10}$. Elaborate functionalities can rationally be tailored with precise molecular scale control through artificial assembly based on judicious selection and coordination of heterogeneous counterparts ${ }^{11-14}$. Intriguing surface effects and physical-chemical properties have gradually been uncovered in $2 \mathrm{D}$ hetero-layered materials owing to their large surface-tovolume ratio and confined thickness at an atomic scale ${ }^{15-18}$. The 2D materials, specifically metal chalcogenides, possess exquisite photo- and electrochemical capabilities that include energy storage batteries/supercapacitors and energy conversion photo/electrocatalysis systems ${ }^{10,19,20}$. Unlike electronic transistor devices, these applications generally require large quantities of $2 \mathrm{D}$ hetero-layered materials. Though a wide variety of $2 \mathrm{D}$ layered semiconductor materials have been exfoliated into individual layers, these layered materials typically undergo large extent of swelling phase induced by intercalator or solvation species ${ }^{21-23}$. Realization of high-volume exfoliation with ultrathin sheet-like crystallite in a facile manner and clean medium still remains scarce. Moreover, the issue of lattice mismatching hinders composition customization of heterostructures owing to the ineffective direct epitaxial growth of some mismatched metal chalcogenide materials. Collectively, all the aforementioned issues limit the scalability of 2D ultrathin hetero-layered metal chalcogenides towards fundamental exploration and advanced functional applications.

Herein, we readily exfoliate the metal chalcogenide semiconductor $\mathrm{ZnIn}_{2} \mathrm{~S}_{4}$ into single-unit-cell layered structure (ca. $2.5 \mathrm{~nm}$ ) via a self-surface charge exfoliation in pure water medium. Successive electrostatic coupling with another transition metal chalcogenide (for example, $\mathrm{MoSe}_{2}$ ) enables construction of arbitrary ultrathin hetero-layered hybrids in a large scale. Such an approach offers salient features, that is, independent thickness and composition control of individual layer assembly, and no constraint by lattice matching prerequisite into functional ultrathin heterostructures. Distinct emission lifetime reduction and photoluminescence quenching of the hetero-layered hybrid ascertain strong interlayer coupling and efficient charge transfer between the components. Surface and interfacial-dominated photocatalysis, a promising strategy for solar energy conversion $^{24-32}$, is adopted to demonstrate the reliability of the catalytically rich $2 \mathrm{D}$ ultrathin $\mathrm{ZnIn}_{2} \mathrm{~S}_{4} / \mathrm{MoSe}_{2}$ hetero-layered material. The as-synthesized $\mathrm{ZnIn}_{2} \mathrm{~S}_{4} / \mathrm{MoSe}_{2}$ concurrently realize efficient separation and transfer of photogenerated charge carriers, acceleration of surface proton reduction with abundant active sites as well as enhanced visible light absorption that circumvent the limitations of conventional photocatalysts ${ }^{33}$. Consequently, the $\mathrm{ZnIn}_{2} \mathrm{~S}_{4} / \mathrm{MoSe}_{2}$ displays high-performance visible-light-driven $\mathrm{H}_{2}$ evolution activity of $6,454 \mu \mathrm{mol} \mathrm{g}^{-1} \mathrm{~h}^{-1}, \sim 15$ and 4 times as high as that of bulk and bare $\mathrm{ZnIn}_{2} \mathrm{~S}_{4}$ nanosheets, respectively. Importantly, the $2 \mathrm{D}$ hetero-layered hybrid shows high stability with prolonged $80 \mathrm{~h}$ cycling and catalytic reactivity retention after storing over a few months that further attests to the integrity of the constructed 2D materials for prospective advanced applications. Furthermore, we test the applicability of this approach on other 2D metal sulfides, namely $\mathrm{CdIn}_{2} \mathrm{~S}_{4}$ and $\mathrm{In}_{2} \mathrm{~S}_{3}$ that also show scalable self-surface charge exfoliation. Similarly, the constructed hetero-layered hybrids of $\mathrm{CdIn}_{2} \mathrm{~S}_{4} / \mathrm{MoSe}_{2}$ and $\mathrm{In}_{2} \mathrm{~S}_{3} / \mathrm{MoSe}_{2}$ feature enhanced photocatalytic activity and stability.

\section{Results}

Self-surface charge exfoliation of ultrathin $\mathrm{ZnIn}_{2} \mathrm{~S}_{4}$ layers. The ultrathin single-unit-cell $\mathrm{ZnIn}_{2} \mathrm{~S}_{4}$ layers were prepared via a facile low-temperature refluxing method, followed by a water-assisted surfactant/intercalator-free exfoliation process, as schematically illustrated in Fig. 1a (for more details, see Methods). Scanning electron microscopy (SEM) images of bulk $\mathrm{ZnIn}_{2} \mathrm{~S}_{4}$ in Fig. $1 \mathrm{~b}$ and Supplementary Fig. 1 reveal a uniform morphology of sheet-like structure. The energy-dispersive X-ray spectroscopy (EDX) (Supplementary Fig. 2) and X-ray diffraction (XRD) (Supplementary Fig. 3) analysis confirm the elemental composition and high purity of the as-synthesized $\mathrm{ZnIn}_{2} \mathrm{~S}_{4}$ with hexagonal phase structure (cell parameters of $a=b=3.85 \AA, c=24.68 \AA$, JCPDS No. 65-2,023).

Notably, the dispersion of $\mathrm{ZnIn}_{2} \mathrm{~S}_{4}$ in deionized (DI) water reveals a strong negatively charged surface with a zeta potential value of $-36.5 \mathrm{mV}$ (Supplementary Fig. 4). This can be ascribed to the presence of excess amount of $S^{2}-$ that has been adsorbed onto the $\mathrm{ZnIn}_{2} \mathrm{~S}_{4}$ surface during the synthesis process ${ }^{34-36}$. Other supporting evidences are appended in Supplementary Figs 5 and 6 . The self-surface charge within the layered nanostructure significantly weakens the interaction between $\mathrm{ZnIn}_{2} \mathrm{~S}_{4}$ interlayers. Owing to Coulombic repulsion, these layers repel each other and are readily exfoliated with the assistance of shear forces triggered by mild sonication in the absence of any intercalator and surfactant species (Supplementary Fig. 7).

Transmission electron microscopy (TEM) images (Fig. 1c and Supplementary Fig. 8) of the as-exfoliated $\mathrm{ZnIn}_{2} \mathrm{~S}_{4}$ layers display $2 \mathrm{D}$ sheet structure with a nearly transparent feature, implying the ultrathin nature of the exfoliated product. This self-surface charge promoted exfoliation can be corroborated by the controlled experiment of ultrasonic exfoliation of $\mathrm{ZnIn}_{2} \mathrm{~S}_{4}$ synthesized from the addition of a stoichiometric amount of $S^{2}-$ (denoted as $\mathrm{ZnIn}_{2} \mathrm{~S}_{4}-\mathrm{S}$, see Methods for more details), as illustrated in Supplementary Fig. 9. The as-obtained $\mathrm{ZnIn}_{2} \mathrm{~S}_{4}-\mathrm{S}$ displays a weak zeta potential of $-5.2 \mathrm{mV}$ (Supplementary Fig. 10). Accordingly, the dispersion of $\mathrm{ZnIn}_{2} \mathrm{~S}_{4}-\mathrm{S}$ can be easily centrifuged after ultrasonication. The TEM image in Supplementary Fig. 11 demonstrates that the $\mathrm{ZnIn}_{2} \mathrm{~S}_{4}-\mathrm{S}$ is composed of aggregated layers. In addition, negatively charged $\mathrm{ZnIn}_{2} \mathrm{~S}_{4}$ surface can also be validated by a layer electrostatic self-assembly demonstration of the $\mathrm{ZnIn}_{2} \mathrm{~S}_{4}$ nanosheets on positively charged 3-aminopropyltriethoxysilane (APTES)-modified glass substrate (Supplementary Figs 12 and 13). Correspondingly, a pale yellow thin film can be observed only for the strong negatively charged $\mathrm{ZnIn}_{2} \mathrm{~S}_{4}$ $(-36.5 \mathrm{mV})$ coating on the glass surface. SEM image reveals uniform coverage/assembly of strong negatively charged $\mathrm{ZnIn}_{2} \mathrm{~S}_{4}$ nanosheets on the positive APTES-glass substrate. Conversely, no obvious adsorption of the weak negatively charged $\mathrm{ZnIn}_{2} \mathrm{~S}_{4}-\mathrm{S}$ $(-5.2 \mathrm{mV})$ has been observed on the glass substrate. Altogether, these findings confirm the existence of strong negatively self-charged $\mathrm{ZnIn}_{2} \mathrm{~S}_{4}$ surface that facilitates facile interlayer exfoliation of ultrathin $\mathrm{ZnIn}_{2} \mathrm{~S}_{4}$ nanosheets without the assistance of surfactant/intercalator additives.

Moreover, the corresponding selected-area electron diffraction pattern in Fig. 1c inset shows clear bright spots that correspond to the hexagonal structure and single-crystalline characteristic of the ultrathin $\mathrm{ZnIn}_{2} \mathrm{~S}_{4}$ nanosheets. In addition, the high-resolution TEM (HRTEM) image in Fig. 1c inset displays distinct lattice fringes of ca. $0.32 \mathrm{~nm}$, corresponding to the (102) crystallographic plane of $\mathrm{ZnIn}_{2} \mathrm{~S}_{4}$. The typical Tyndall effect observed for the as-exfoliated $\mathrm{ZnIn}_{2} \mathrm{~S}_{4}$ suspension using a red laser (Fig. 1b, inset) indicates the formation of freestanding and highly dispersed ultrathin $\mathrm{ZnIn}_{2} \mathrm{~S}_{4}$ layers. More importantly, the colloid ultrathin $\mathrm{ZnIn}_{2} \mathrm{~S}_{4}$ nanosheets can be easily exfoliated into large scale (Supplementary Fig. 14) that is essential for further utilization.

TEM characterization cannot unambiguously determine the ultimate thickness of the layers. In this context, atomic force microscopy (AFM) is used to provide an estimated quantitative layer thickness. As shown in Fig. 1d, the topography of the 
a
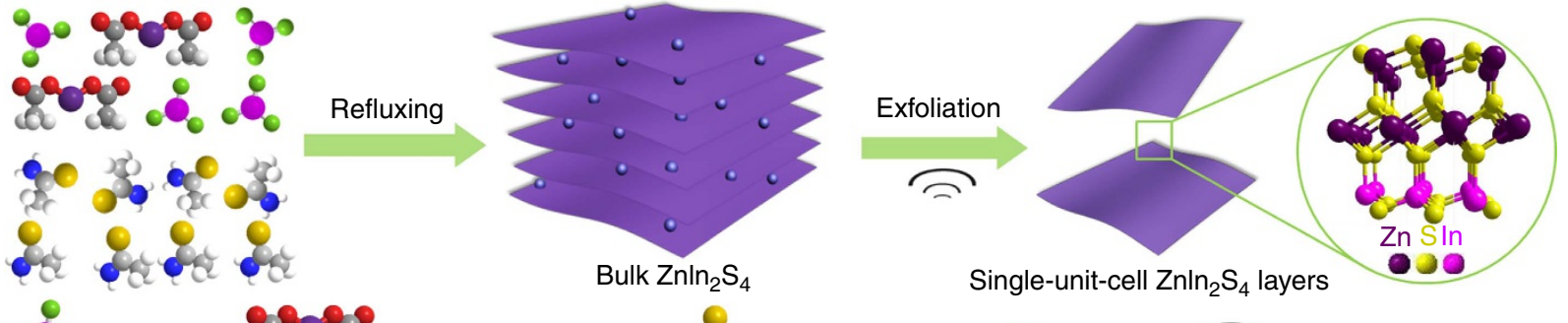

$\mathrm{O}_{0} \mathrm{InCl}_{3}$

Q⿱⺈⿵⺆⿻二丨冂口. $\mathrm{Zn}\left(\mathrm{OOCCH}_{3}\right)_{2}$

eo $\mathrm{C}_{2} \mathrm{H}_{5} \mathrm{NS}$

$\mathrm{H}_{2} \mathrm{O}$

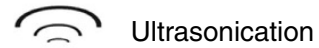

b

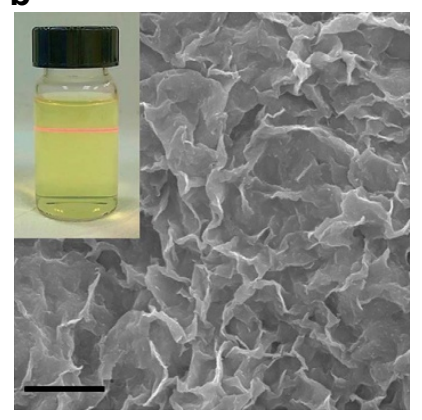

c

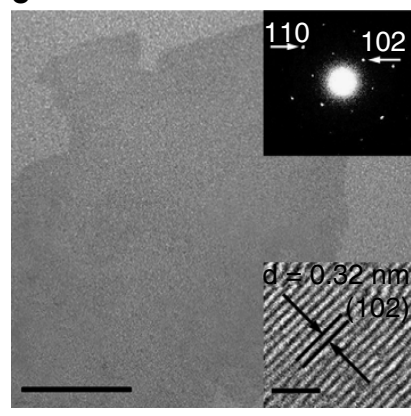

d

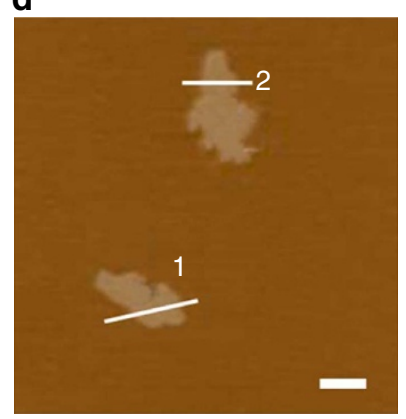

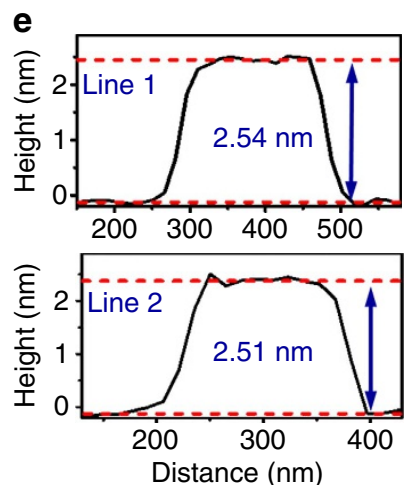

Figure 1 | Schematic illustration of the synthesis of single-unit-cell $\mathbf{Z n} \mathbf{l} \mathbf{n}_{\mathbf{2}} \mathbf{S}_{\mathbf{4}}$ layers and characterization. (a) A self-surface charge promoted exfoliation of clean and freestanding single-unit-cell $Z \mathrm{Znn}_{2} \mathrm{~S}_{4}$ layers in surfactant/intercalator-free medium. (b) Scanning electron microscopy (SEM) image of bulk $\mathrm{ZnIn}_{2} \mathrm{~S}_{4}$. Scale bar, $2 \mu \mathrm{m}$ (c) Transmission electron microscopy (TEM) image of single-unit-cell $\mathrm{Znln}_{2} \mathrm{~S}_{4}$ layers. Scale bar, $50 \mathrm{~nm}$. (d) Atomic force microscopy (AFM) image and (e) corresponding height images of single-unit-cell $\mathrm{ZnIn}_{2} \mathrm{~S}_{4}$ layers. Scale bar, $200 \mathrm{~nm}$. The inset in $\mathbf{b}$ is photograph of Tyndall effect of the $\mathrm{ZnIn}_{2} \mathrm{~S}_{4}$ suspension; insets in c are the corresponding selected-area electron diffraction (SAED) pattern and high-resolution TEM (HRTEM) image of single-unit-cell $\mathrm{ZnIn}_{2} \mathrm{~S}_{4}$ layers.

as-prepared $\mathrm{ZnIn}_{2} \mathrm{~S}_{4}$ presents $2 \mathrm{D}$ structure with smooth surface. The corresponding height profiles in Fig. 1e show that the typical thickness of the as-exfoliated layers is $\sim 2.5 \mathrm{~nm}$, validating the ultrathin nature of the $\mathrm{ZnIn}_{2} \mathrm{~S}_{4}$. Considering that the $c$ parameter of $\mathrm{ZnIn}_{2} \mathrm{~S}_{4}$ is $24.68 \AA$ (Supplementary Fig. 15), the thickness of $\mathrm{ZnIn}_{2} \mathrm{~S}_{4}$ is well in agreement with the thickness of a unit cell along the [001] axis ${ }^{37}$. Thus, it is reasonable to deduce that each ultrathin $\mathrm{ZnIn}_{2} \mathrm{~S}_{4}$ nanosheet with a thickness of ca. $2.5 \mathrm{~nm}$ is a single-unit-cell $\mathrm{ZnIn}_{2} \mathrm{~S}_{4}$ atomic layer. According to the diffusion formula of $t=d^{2} / k^{2} D$ ( $d$ is the particle size, $k$ is a constant, $D$ is the diffusion coefficient of electron-hole pairs $)^{5,7}$, the ultrathin single-unit-cell $\mathrm{ZnIn}_{2} \mathrm{~S}_{4}$ atomic layer will significantly shorten the diffusion length and time of charge carriers taken to reach the surface, enable the photoexcited electron-hole pairs to transport from the interior to the surface fast, and thus lead to higher charge separation efficiency of the ultrathin $\mathrm{ZnIn}_{2} \mathrm{~S}_{4}$ than its counterpart of bulk $\mathrm{ZnIn}_{2} \mathrm{~S}_{4}$, as verified by the photocurrent and photoluminescence (PL) analysis in Supplementary Figs 16 and 17.

Generalized synthesis of ultrathin $\operatorname{CdIn}_{2} S_{4}$ and $\operatorname{In}_{2} S_{3}$ layers. The suitability of self-surface charge exfoliation as a general approach for large-scale preparation of other 2D metal sulfides, that is, $\mathrm{CdIn}_{2} \mathrm{~S}_{4}$ and $\operatorname{In}_{2} \mathrm{~S}_{3}$, has also been demonstrated. As shown in Supplementary Fig. 18, the $\mathrm{CdIn}_{2} \mathrm{~S}_{4}$ dispersion in DI water shows a strong negative charge with a zeta potential value of $-39.7 \mathrm{mV}$. TEM images in Supplementary Fig. 19 demonstrate the $2 \mathrm{D}$ sheet structure of the exfoliated $\mathrm{Cd} \operatorname{In}_{2} \mathrm{~S}_{4}$ nanosheets without using any surfactant or intercalator under moderate ultrasonication. The inset in Supplementary Fig. 19 shows HRTEM image where the distinct lattice fringes of $0.33 \mathrm{~nm}$ correspond to the (311) crystallographic plane of $\mathrm{CdIn}_{2} \mathrm{~S}_{4}$. AFM topography reveals the ultrathin structure of the exfoliated $\mathrm{CdIn}_{2} \mathrm{~S}_{4}$ with the thickness of $\sim 2.3 \mathrm{~nm}$ (Supplementary Fig. 20). Typical Tyndall effect is observed for the as-exfoliated CdIn $\mathrm{S}_{4}$ suspension (Supplementary Fig. 21), indicating the formation of large-scale freestanding and highly dispersed $\mathrm{CdIn}_{2} \mathrm{~S}_{4}$ layers. Moreover, the respective $\mathrm{EDX}$ and XRD analyses in Supplementary Figs 22 and 23 confirm the as-synthesized $\mathrm{CdIn}_{2} \mathrm{~S}_{4}$ nanosheets with cubic phase structure (JCPDS No. 27-0060).

Likewise, this self-surface charge strategy is also exploited to exfoliate $\mathrm{In}_{2} \mathrm{~S}_{3}$ into a large-scale colloidal dispersion of ultrathin nanosheets (Supplementary Fig. 24). Zeta potential measurement displays a strong negatively charged surface (zeta potential of $-41.9 \mathrm{mV}$ ) of $\mathrm{In}_{2} \mathrm{~S}_{3}$ (Supplementary Fig. 25). TEM images in Supplementary Fig. 26 show 2D sheet structure with distinct lattice fringes (ca. $0.32 \mathrm{~nm}$ ) of $\mathrm{In}_{2} \mathrm{~S}_{3}$ (311) crystallographic plane. The thickness of the obtained $\operatorname{In}_{2} \mathrm{~S}_{3}$ is $\sim 4.5 \mathrm{~nm}$ (Supplementary Fig. 27). Moreover, EDX result confirms the elemental composition of $\operatorname{In}_{2} S_{3}$ and XRD pattern reveals the pure cubic phase of the synthesized $\mathrm{In}_{2} \mathrm{~S}_{3}$ (JCPDS No. 65-0459) (Supplementary Figs 28 and 29).

Construction of hetero-layered hybrids. Recent advances in creating heterostructures based on 2D atomic crystals by artificial combination of various $2 \mathrm{D}$ materials have shown to strongly modulate electronic and optical properties ${ }^{17}$. By virtue of the 2D configuration with large surface area, along with its complementary high interfacial contact with other components, the single-unit-cell $\mathrm{ZnIn}_{2} \mathrm{~S}_{4}$ layers provide a favourable platform for the fabrication of hybrid composite ${ }^{17,38}$. In this context, $\mathrm{MoSe}_{2}$ nanosheets are integrated with the ultrathin $\mathrm{ZnIn}_{2} \mathrm{~S}_{4}$ via a surface charge promoted self-assembly method that is driven by 
strong electrostatic attraction between the negatively charged $\mathrm{ZnIn}_{2} \mathrm{~S}_{4}$ layers and positively charged $\mathrm{MoSe}_{2}$ (see Methods for more details). The electrostatic self-assembly method efficiently circumvents the requirement of lattice matching of two individual layers for assembling hetero-layer structure ${ }^{39,40}$. Consequently, a large quantity of hetero-layered $\mathrm{ZnIn}_{2} \mathrm{~S}_{4} / \mathrm{MoSe}_{2}$ can be facilely obtained (Supplementary Fig. 30). Moreover, this method also imparts strong hetero-interlayer coupling that effectively promotes interfacial charge carriers transfer ${ }^{4-43}$ that will be discussed later.

Figure 2a,b shows the TEM images of $\mathrm{MoSe}_{2}$, indicating that the sheets are typically composed of 2-4 layers with an interlayer spacing of $0.65 \mathrm{~nm}$ that corresponds to the (002) plane of hexagonal $\mathrm{MoSe}_{2}$ (refs 44,45). The HRTEM image in Fig. 2c shows a $d$ spacing of $0.28 \mathrm{~nm}$ that matches with the interspacing of (100) $\mathrm{MoSe}_{2}$ (refs 46,47). After the integration of $\mathrm{MoSe}_{2}$ with the single-unit-cell $\mathrm{ZnIn}_{2} \mathrm{~S}_{4}$ layers, the as-prepared $\mathrm{ZnIn}_{2} \mathrm{~S}_{4} / \mathrm{MoSe}_{2}$ yields a hetero-layered structure (Fig. 2d,e). Figure $2 \mathrm{f}$ and Supplementary Fig. 31 show HRTEM images of $\mathrm{ZnIn}_{2} \mathrm{~S}_{4} / \mathrm{MoSe}_{2}$ that display interlayer spacing of $\mathrm{MoSe}_{2}$ $(0.65 \mathrm{~nm})$ and distinct lattice fringes of $\mathrm{ZnIn}_{2} \mathrm{~S}_{4}(0.32 \mathrm{~nm})$, confirming the co-existence and interfacial contact of the two components. In addition, Supplementary Fig. 32 shows overlapping TEM mapping of various elements, also indicating the lamellar structure formation of vertically coordinated $\mathrm{ZnIn}_{2} \mathrm{~S}_{4}$ and $\mathrm{MoSe}_{2}$ heterostructure. The corresponding EDX spectrum (Fig. 3a) shows the coexistence of $\mathrm{Zn}, \mathrm{In}, \mathrm{S}, \mathrm{Mo}$ and Se elements, whereas the EDX mapping (Fig. 3b) demonstrates the homogeneous distribution of these elements throughout the $\mathrm{ZnIn}_{2} \mathrm{~S}_{4} / \mathrm{MoSe}_{2}$ composite. Therefore, based on the above analyses, it is reasonable to infer that the ultrathin single-unitcell $\mathrm{ZnIn}_{2} \mathrm{~S}_{4}$ layers are intimately coupled with the layered $\mathrm{MoSe}_{2}$ featuring a sheet-on-sheet hetero-layer structure, as schematically reflected in Fig. 3c.

Moreover, the XRD patterns of $\mathrm{ZnIn}_{2} \mathrm{~S}_{4}$ and $\mathrm{ZnIn}_{2} \mathrm{~S}_{4} / \mathrm{MoSe}_{2}$ composite (Supplementary Fig. 33) present analogous diffraction peaks of hexagonal phase $\mathrm{ZnIn}_{2} \mathrm{~S}_{4}$. The absence of typical $\mathrm{MoSe}_{2}$ peaks (Supplementary Fig. 34) could be ascribed to the relatively low diffraction intensity of $\mathrm{MoSe}_{2}$ peaks shielded by the strong and broad peaks of $\mathrm{ZnIn}_{2} \mathrm{~S}_{4}$. Notably, Fig. 3d shows the Raman spectra of $\mathrm{ZnIn}_{2} \mathrm{~S}_{4}$ and $\mathrm{ZnIn}_{2} \mathrm{~S}_{4} / \mathrm{MoSe}_{2}$ where a characteristic peak at $240 \mathrm{~cm}^{-1}$ corresponding to the ' $\mathrm{A}_{1 \mathrm{~g}}$ ' band of $\mathrm{MoSe}_{2}$ (refs 44,48,49) (Supplementary Fig. 35) is observed for $\mathrm{ZnIn}_{2} \mathrm{~S}_{4} /$ $\mathrm{MoSe}_{2}$ sample, confirming the formation of a composite with $\mathrm{MoSe}_{2}$ in the matrix of $\mathrm{ZnIn}_{2} \mathrm{~S}_{4}$. In addition, the shift of the ' $\mathrm{A}_{1 \mathrm{~g}}$ ' band of $\mathrm{ZnIn}_{2} \mathrm{~S}_{4} / \mathrm{MoSe}_{2}$ as compared with that of bare $\mathrm{MoSe}_{2}$ $\left(241.1 \mathrm{~cm}^{-1}\right)$ also implies the reduced layer aggregation of $\mathrm{MoSe}_{2}$ nanosheets in the hybrid composite ${ }^{48,49}$. The ultraviolet-visible (UV-vis) absorption spectra in Supplementary Fig. 36 show that the $\mathrm{ZnIn}_{2} \mathrm{~S}_{4} / \mathrm{MoSe}_{2}$ displays enhancement in visible-light absorption as compared with $\mathrm{ZnIn}_{2} \mathrm{~S}_{4}$. This can be attributed to the intrinsic background absorption of black-coloured $\mathrm{MoSe}_{2}$ (Supplementary Fig. 37). To further determine the composition and chemical states of the composite, the $\mathrm{ZnIn}_{2} \mathrm{~S}_{4} / \mathrm{MoSe}_{2}$ has been characterized by X-ray photoelectron spectroscopy (XPS). The doublet peaks for Mo $3 d$ at 228.7 and $231.9 \mathrm{eV}$ (top panel of Fig. $3 \mathrm{e}$ ) can be assigned to the $\mathrm{Mo}^{+4}$ valence state, whereas the peak at $226.4 \mathrm{eV}$ should be assigned to S $2 s$ (ref. 15). In the Se $3 d$ XPS spectrum (bottom panel of Fig. 3e), the peaks at 54.4 and $55.3 \mathrm{eV}$ are ascribed to $\mathrm{Se}^{2-}$ (ref. 47). Meanwhile, the highresolution XPS spectra of $\mathrm{Zn} 2 p$ peaks at 1021.8 and $1044.8 \mathrm{eV}$, In $3 d$ peaks at 445.0 and $452.6 \mathrm{eV}$ and $\mathrm{S} 2 p$ at 161.9 and $163.0 \mathrm{eV}$ (Supplementary Fig. 38) can be assigned to $\mathrm{Zn}^{2+}, \mathrm{In}^{3+}$ and $\mathrm{S}^{2-}$ of $\mathrm{ZnIn}_{2} \mathrm{~S}_{4}$, respectively ${ }^{50-52}$. The XPS analysis corroborates the presence of $\mathrm{MoSe}_{2}$ in the $\mathrm{ZnIn}_{2} \mathrm{~S}_{4} / \mathrm{MoSe}_{2}$ composite.

Photoelectrochemical properties. It has been well accepted that layered transition metal chalcogenides with exposed edge sites can effectively decrease activation energy/overpotential of redox reaction ${ }^{17}$ that are desirable for photo-/electrocatalytic processes. According to the energy band structures of $\mathrm{ZnIn}_{2} \mathrm{~S}_{4}$ and $\mathrm{MoSe}_{2}$, the photogenerated electrons from the excitation of $\mathrm{ZnIn}_{2} \mathrm{~S}_{4}$ are thermodynamically available for transferring to $\mathrm{MoSe}_{2}$. Therefore, it is anticipated that the $\mathrm{ZnIn}_{2} \mathrm{~S}_{4} / \mathrm{MoSe}_{2}$ hetero- a

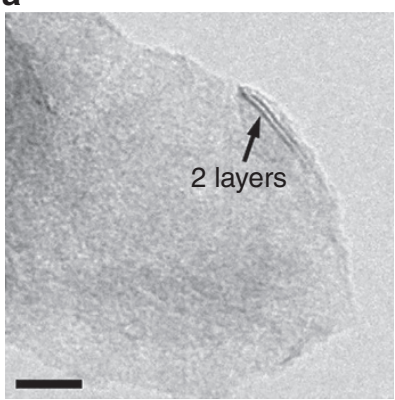

d

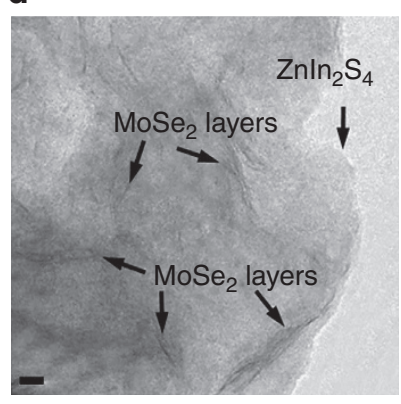

b

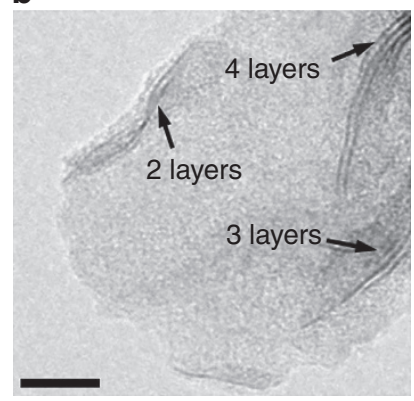

e

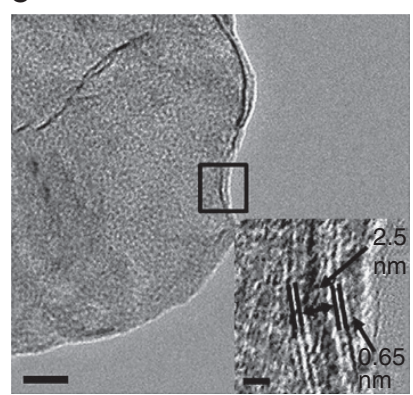

c

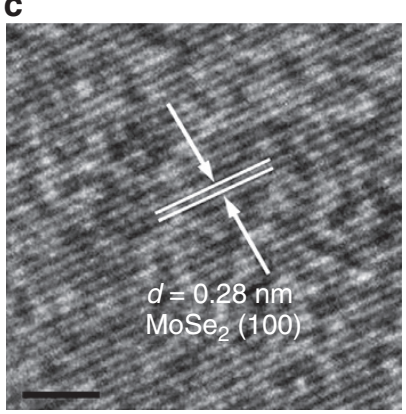

f

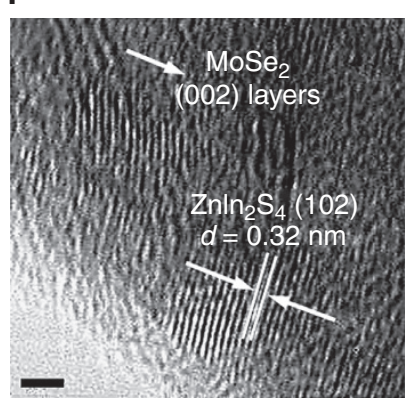

Figure 2 | Morphology and structure of few-layered $\mathbf{M o S e}_{\mathbf{2}}$ and hetero-layered $\mathbf{Z n} \mathbf{I n}_{\mathbf{2}} \mathbf{S}_{\mathbf{4}} / \mathbf{M o S e}_{\mathbf{2}}$. (a,b,d,e) Transmission electron microscopy (TEM) images of few-layered $\mathrm{MoSe}_{2}(\mathbf{a}, \mathbf{b})$ and hetero-layered $\mathrm{ZnIn}_{2} \mathrm{~S}_{4} / \mathrm{MoSe}_{2}(\mathbf{d}, \mathbf{e})$. Scale bar, $10 \mathrm{~nm}$. (c,f) High-resolution TEM (HRTEM) images of MoSe 2 (c) and $\mathrm{ZnIn}_{2} \mathrm{~S}_{4} / \mathrm{MoSe}_{2}$ (f). Scale bar, $2 \mathrm{~nm}$. The inset in $\mathbf{e}$ is the magnification of the image shown in the black box of Fig. $2 \mathrm{e}$. Scale bar, $2 \mathrm{~nm}$. 
a

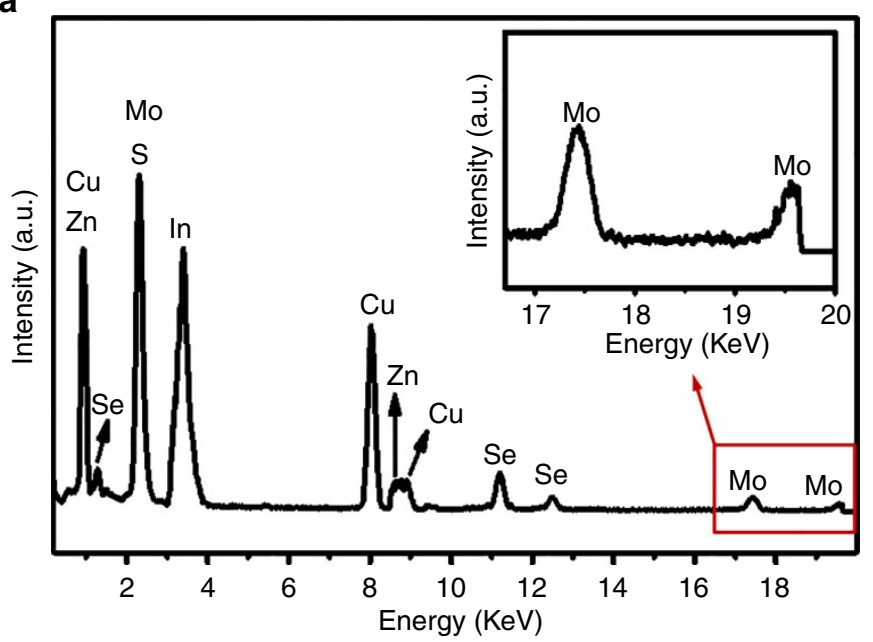

b

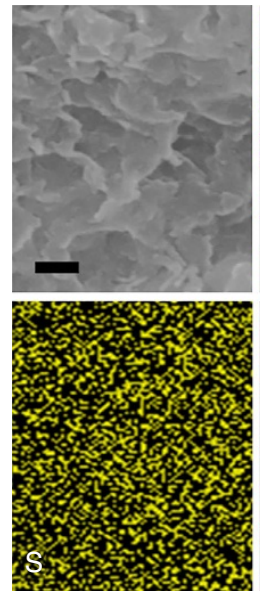

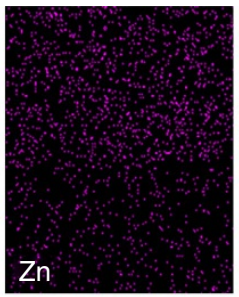
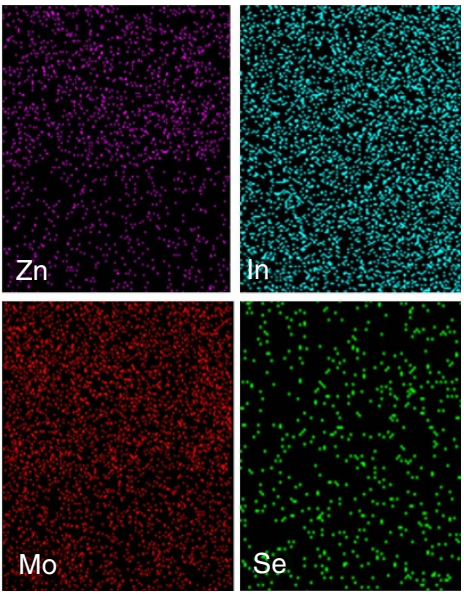

C

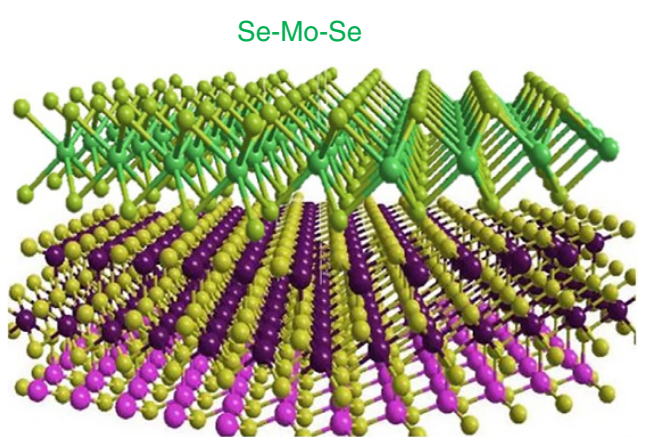

S-Zn-S-In-S-In-S d

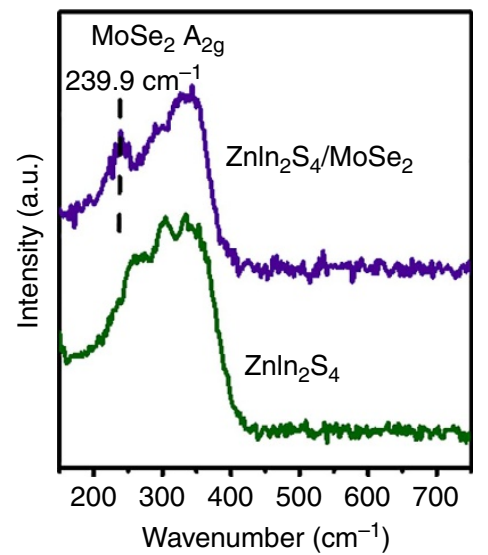

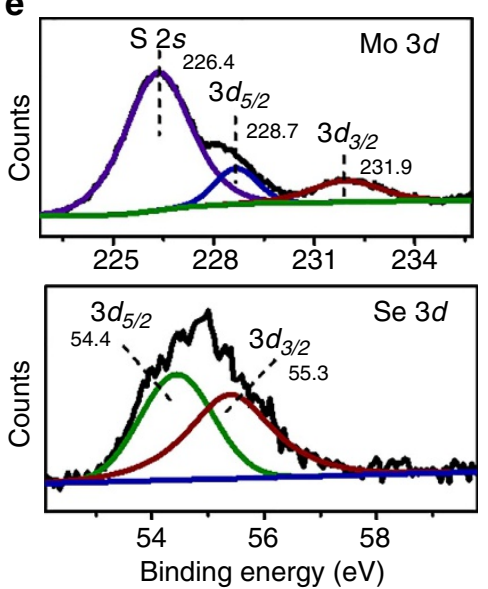

Figure 3 | Characterization of hetero-layered $\mathbf{Z n I n} \mathbf{n}_{\mathbf{4}} / \mathbf{M o S e}_{\mathbf{2}}$. (a) Energy-dispersive X-ray (EDX) spectrum and (b) mapping images of the as-prepared $\mathrm{ZnIn}_{2} \mathrm{~S}_{4} / \mathrm{MoSe}_{2}$. Scale bar, $200 \mathrm{~nm}$. (c) Schematic illustration of the sheet-on-sheet $\mathrm{ZnIn} \mathrm{S}_{4} / \mathrm{MoSe}_{2}$ hetero-layer structure. (d) Raman spectra of $\mathrm{ZnIn}_{2} \mathrm{~S}_{4}$ and $\mathrm{ZnIn}_{2} \mathrm{~S}_{4} / \mathrm{MoSe}_{2}$. (e) High-resolution X-ray photoelectron spectroscopy (XPS) spectra of Mo 3d and Se 3d.

layered structure not only promotes charge separation and transport driven by junction/interface between the $\mathrm{MoSe}_{2}$ and the light harvester $\mathrm{ZnIn}_{2} \mathrm{~S}_{4}$, but also facilitates the proton reduction on the surface of $\mathrm{MoSe}_{2}$. To gain more insight into its optoelectronic properties, a series of complementary photo- and electrochemical characterizations were carried out. Figure $4 \mathrm{a}$ displays the linear sweep voltammetry curves of $\mathrm{ZnIn}_{2} \mathrm{~S}_{4} / \mathrm{MoSe}_{2}$ composite and bulk $\mathrm{ZnIn}_{2} \mathrm{~S}_{4}$, revealing a higher cathodic current density that is attributed to the reduction of water to $\mathrm{H}_{2}$ (ref. 53) over hetero-layered $\mathrm{ZnIn}_{2} \mathrm{~S}_{4} / \mathrm{MoSe}_{2}$ than bulk $\mathrm{ZnIn}_{2} \mathrm{~S}_{4}$. Simultaneously, the controlled experiment over bare fluoride tin oxide (FTO) shows no obvious cathodic current under the same potential range (Supplementary Fig. 39). The result indicates that the integration of $\mathrm{MoSe}_{2}$ with $\mathrm{ZnIn}_{2} \mathrm{~S}_{4}$ accelerates the protonation and subsequent $\mathrm{H}_{2}$ formation rate of $\mathrm{ZnIn}_{2} \mathrm{~S}_{4} / \mathrm{MoSe}_{2}$ as compared with that of bulk $\mathrm{ZnIn}_{2} \mathrm{~S}_{4}$.

In addition, Fig. $4 \mathrm{~b}$ displays the time-resolved photoluminescence spectra of bulk $\mathrm{ZnIn}_{2} \mathrm{~S}_{4}$ and hetero-layer structured $\mathrm{ZnIn}_{2} \mathrm{~S}_{4} / \mathrm{MoSe}_{2}$, which probe the specific charge carrier dynamics of nanosystems ${ }^{54,55}$. The emission decay curves of the samples are fitted by biexponential kinetics function (Supplementary Note 1, Supplementary Equation 1) in which two decay components are derived (insets in Fig. 4b) $\left(\tau_{1}\right.$ is originated from the nonradiative recombination of charge carriers in the defect states of $\mathrm{ZnIn}_{2} \mathrm{~S}_{4}$, whereas the longer lifetime component of $\tau_{2}$ is caused by the recombination of free excitons in the $\mathrm{ZnIn}_{2} \mathrm{~S}_{4}$ ). For $\mathrm{ZnIn}_{2} \mathrm{~S}_{4} / \mathrm{MoSe}_{2}$, the emission lifetimes of both components ( $\left.\tau_{1}=0.31 \mathrm{~ns}, \quad \tau_{2}=0.38 \mathrm{~ns}\right)$ are shorter than that of the corresponding bulk $\mathrm{ZnIn}_{2} \mathrm{~S}_{4}$ counterpart $\left(\tau_{1}=0.73 \mathrm{~ns}\right.$, $\tau_{2}=3.77 \mathrm{~ns}$ ). The average emission lifetime (calculated from Supplementary Note 1, Supplementary Equation 2), which reflects the overall emission decay behaviour of sample, has also displayed an obvious decrease for $\mathrm{ZnIn}_{2} \mathrm{~S}_{4} / \mathrm{MoSe}_{2}(2.43 \mathrm{~ns})$ as compared with that of bulk $\mathrm{ZnIn}_{2} \mathrm{~S}_{4}$ (3.78 ns). Meanwhile, the steady-state PL spectra in Fig. $4 \mathrm{~d}$ show obvious PL quenching of hetero-layered $\mathrm{ZnIn}_{2} \mathrm{~S}_{4} / \mathrm{MoSe}_{2}$ hybrid. The corresponding observations of PL quenching and lifetime reduction suggest the establishment of an electron transfer channel from $\mathrm{ZnIn}_{2} \mathrm{~S}_{4}$ to $\mathrm{MoSe}_{2}$ in a nonradiative quenching pathway (Fig. 4c) ${ }^{54,55}$. Accordingly, this leads to efficient interfacial charge transfer and suppression of photoexcited charge recombination in the hetero-layered $\mathrm{ZnIn}_{2} \mathrm{~S}_{4} / \mathrm{MoSe}_{2}$ structure.

Furthermore, the electrochemical impedance spectrum of $\mathrm{ZnIn}_{2} \mathrm{~S}_{4} / \mathrm{MoSe}_{2}$ (Fig. 4e) shows a smaller semicircular in the Nyquist plot than that of bare $\mathrm{ZnIn}_{2} \mathrm{~S}_{4}$ nanosheets, indicating a lower charge-transfer resistance in the hybrid composite that warrants efficient transportation and separation of charge carriers $^{56-59}$. As shown in Fig. 4f, the $\mathrm{ZnIn}_{2} \mathrm{~S}_{4} / \mathrm{MoSe}_{2}$ displays obvious transient photocurrent response under visible light irradiation. The current density is comparable to recently 
a

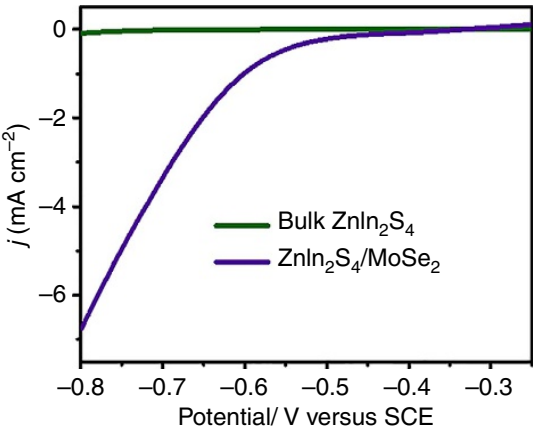

d

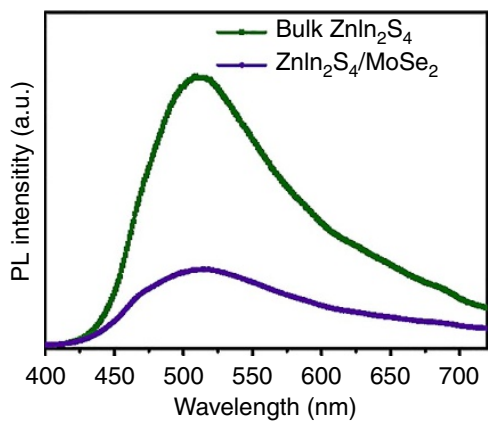

b

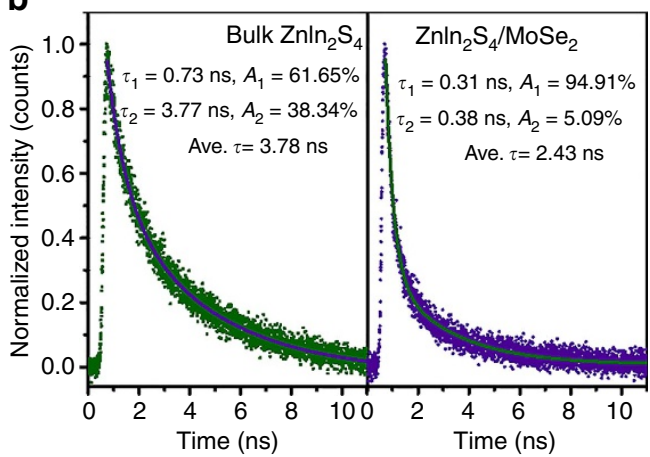

C

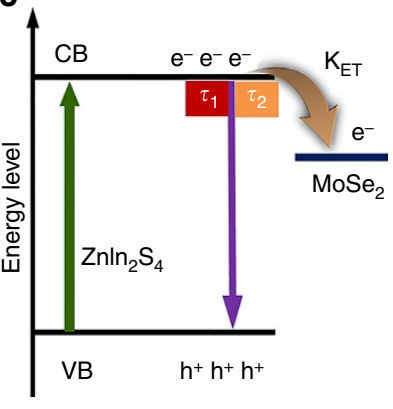

e

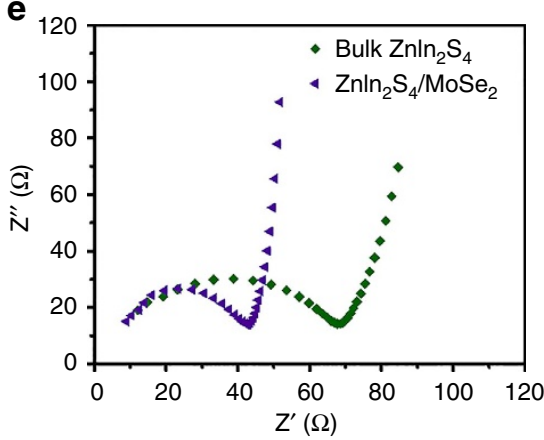

f

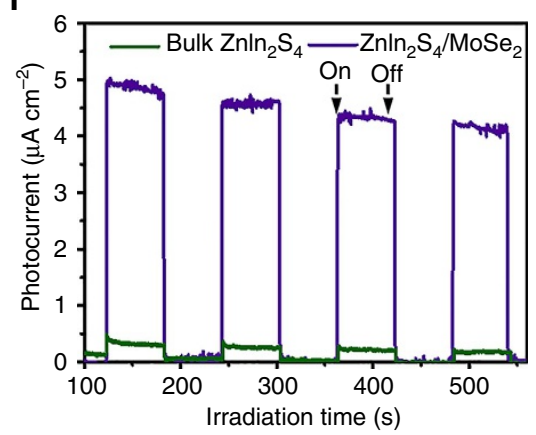

Figure 4 | Photoelectrochemical properties. (a) Linear sweep voltammetry (LSV) curves, (b) time-resolved transient photoluminescence (PL) decay (excitation at $400 \mathrm{~nm}$ and emission at $495 \mathrm{~nm}$ ), (c) schematic illustration of the interfacial charge carrier transfer, (d) steady-state PL spectra, (e) electrochemical impedance spectroscopy Nyquist plots and (f) transient photocurrent responses of bulk $\mathrm{ZnIn}_{2} \mathrm{~S}_{4}$ and hetero-layered $\mathrm{ZnIn} \mathrm{S}_{4} / \mathrm{MoSe}_{2}$ composite. CB, conduction band; VB, valence band.

reported 2D-based photocatalyst systems (Supplementary Table 1). In addition, it is notable that the $\mathrm{ZnIn}_{2} \mathrm{~S}_{4} / \mathrm{MoSe}_{2}$ displays ca. 22-fold photocurrent enhancement as compared with bulk $\mathrm{ZnIn}_{2} \mathrm{~S}_{4}$ under the same experimental condition, and this is a marked improvement that suggests the structure and composition advantage of the hetero-layered $\mathrm{ZnIn}_{2} \mathrm{~S}_{4} / \mathrm{MoSe}_{2}$ composite in promoting the separation and transportation of photogenerated charge carriers. Collectively, these commendable photo- and electrochemical properties support efficient electron-hole pair separation and high surface reaction rate of the $\mathrm{ZnIn}_{2} \mathrm{~S}_{4} / \mathrm{MoSe}_{2}$ hetero-layered structure.

Photocatalytic $\mathbf{H}_{2}$ production performance. Recent experimental results and theoretical predictions suggest that metal chalcogenides are a class of promising inexpensive, earthabundant and visible responsive catalyst alternatives. However, nonoptimal interfacial contact and bulk metal chalcogenides structure limit catalytic activity owing to poor electronic coupling effect and low active sites exposure. Correspondingly, the constructed metal chalcogenide heterostructure that is thinned down to a few layers with incorporated electrostatic coupling is evaluated by photocatalytic $\mathrm{H}_{2}$ production. The photocatalytic performance provides a useful and explicit evaluation to assert the structural integrity and stability of the $2 \mathrm{D}$ hetero-layered structure.

The photocatalytic activity, $\mathrm{H}_{2}$ generation of the ultrathin $\mathrm{ZnIn}_{2} \mathrm{~S}_{4}$ layers and $\mathrm{ZnIn}_{2} \mathrm{~S}_{4} / \mathrm{MoSe}_{2}$ hetero-layered nanohybrids was performed under visible light irradiation $(\lambda>400 \mathrm{~nm})$ using lactic acid as the hole scavenger. As shown in Fig. 5a, the single-unit-cell $\mathrm{ZnIn}_{2} \mathrm{~S}_{4}$ layers show a $\mathrm{H}_{2}$ evolution rate of $\sim 1,748 \mu \mathrm{molg}^{-1} \mathrm{~h}^{-1}$ that is fourfold of the pristine bulk $\mathrm{ZnIn}_{2} \mathrm{~S}_{4}\left(446 \mu \mathrm{molg}^{-1} \mathrm{~h}^{-1}\right)$, indicating enhanced activity of the ultrathin layers. In addition, the photoactivity of the ultrathin
$\mathrm{ZnIn}_{2} \mathrm{~S}_{4}$ is also demonstrated to be much higher than the $\mathrm{H}_{2}$ generation rate of the hydrothermal synthesized $\mathrm{ZnIn}_{2} \mathrm{~S}_{4}$ nanoflowers (Supplementary Fig. 40) of ca. $260 \mu \mathrm{molg}^{-} \mathrm{h}^{-1}$. The augmented photoactivity of $\mathrm{ZnIn}_{2} \mathrm{~S}_{4}$ nanosheets can be attributed to its unique $2 \mathrm{D}$ ultrathin structure that lowers charge-transfer resistance and shortens the diffusion pathway of charge carriers, thus favouring the fast and efficient separation of photogenerated charge carriers (Supplementary Figs 16 and 17).

Furthermore, significant improvement in $\mathrm{H}_{2}$ generation was established after coupling of $\mathrm{MoSe}_{2}$ layers to the ultrathin $\mathrm{ZnIn}_{2} \mathrm{~S}_{4}$ nanosheets forming hetero-layered composites. The amount of $\mathrm{H}_{2}$ evolved increases with $\mathrm{MoSe}_{2}$ content up to $1 \%$ (Fig. 5b). The as-obtained $\mathrm{ZnIn}_{2} \mathrm{~S}_{4} / 1 \% \mathrm{MoSe}_{2}$ displays the highest $\mathrm{H}_{2}$ evolution rate of $6,454 \mu \mathrm{molg}^{-1} \mathrm{~h}^{-1}$, that is $\sim 15$ and 4 times as high as that of bulk $\mathrm{ZnIn}_{2} \mathrm{~S}_{4}$ and $\mathrm{ZnIn}_{2} \mathrm{~S}_{4}$ nanosheets, respectively. Notably, the photoactivity is considerable higher than that of the reference photocatalysts, that is, $\mathrm{ZnIn}_{2} \mathrm{~S}_{4} / 1 \% \mathrm{Pt}$ $\left(4,353 \mu \mathrm{molg}^{-1} \mathrm{~h}^{-1}\right)$ and $\mathrm{ZnIn}_{2} \mathrm{~S}_{4} / 1 \% \mathrm{MoS}_{2}\left(3,860 \mu \mathrm{mol} \mathrm{g}^{-1} \mathrm{~h}^{-1}\right)$, as shown in Fig. $5 \mathrm{c}, \mathrm{d}$. Pt and $\mathrm{MoS}_{2}$ are two exemplary co-catalysts that have been used in reported literature for photocatalytic $\mathrm{H}_{2}$ evolution. The result highlights the effectiveness of $\mathrm{MoSe}_{2}$ that surpasses the classic Pt and $\mathrm{MoS}_{2}$, as a co-catalyst in promoting photocatalytic $\mathrm{H}_{2}$ evolution ${ }^{44,60}$. Importantly, the hetero-layered $\mathrm{ZnIn}_{2} \mathrm{~S}_{4} / \mathrm{MoSe}_{2}$ displays good stability. The cycling test over the optimal $\mathrm{ZnIn}_{2} \mathrm{~S}_{4} / 1 \% \mathrm{MoSe}_{2}$ (Fig. 5e) shows negligible photoactivity loss after 20 consecutive cycles with accumulatively $80 \mathrm{~h}$ under visible light irradiation. Moreover, after storing in ambient conditions for 3 months, the $\mathrm{ZnIn}_{2} \mathrm{~S}_{4} / 1 \% \mathrm{MoSe}_{2}$ retains a high photoactivity as that of the fresh sample (Supplementary Fig. 41). The high photoreactivity and stability of the $2 \mathrm{D}$ hetero-layer $\mathrm{ZnIn}_{2} \mathrm{~S}_{4} / 1 \% \mathrm{MoSe}_{2}$ hybrid provide direct evidence of the large exposed active surface and strong electronic coupling between the interlayers. 
a

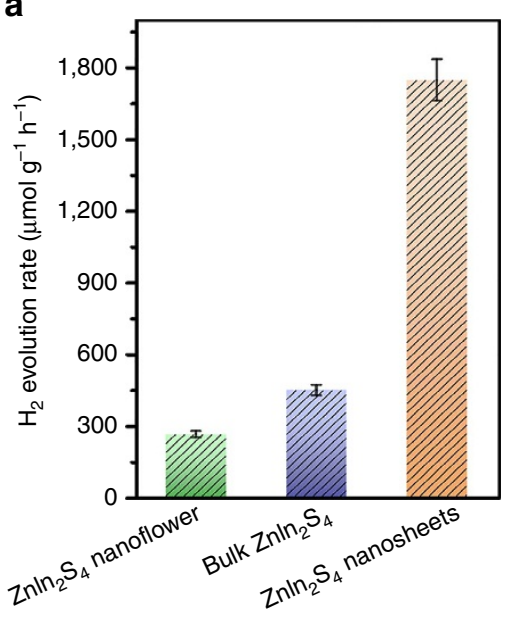

b

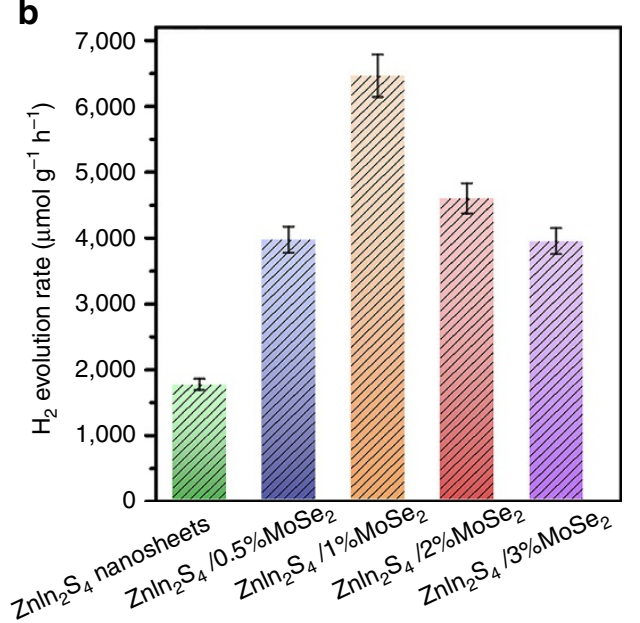

C

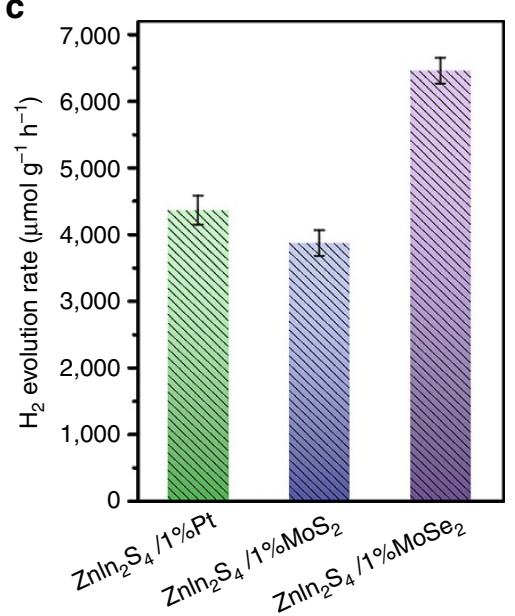

d

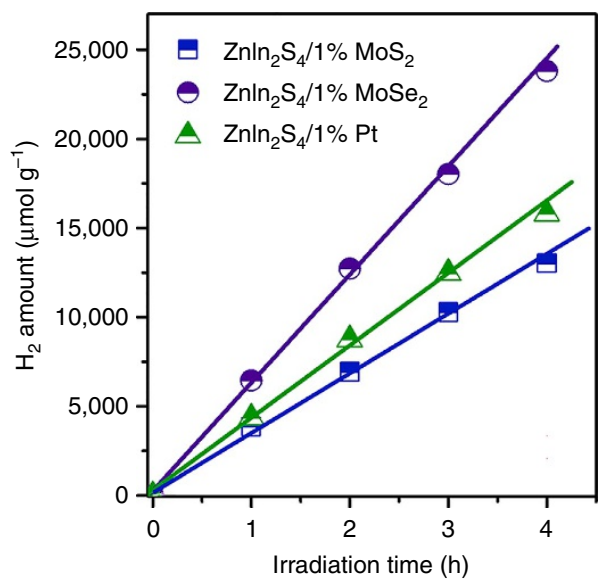

e

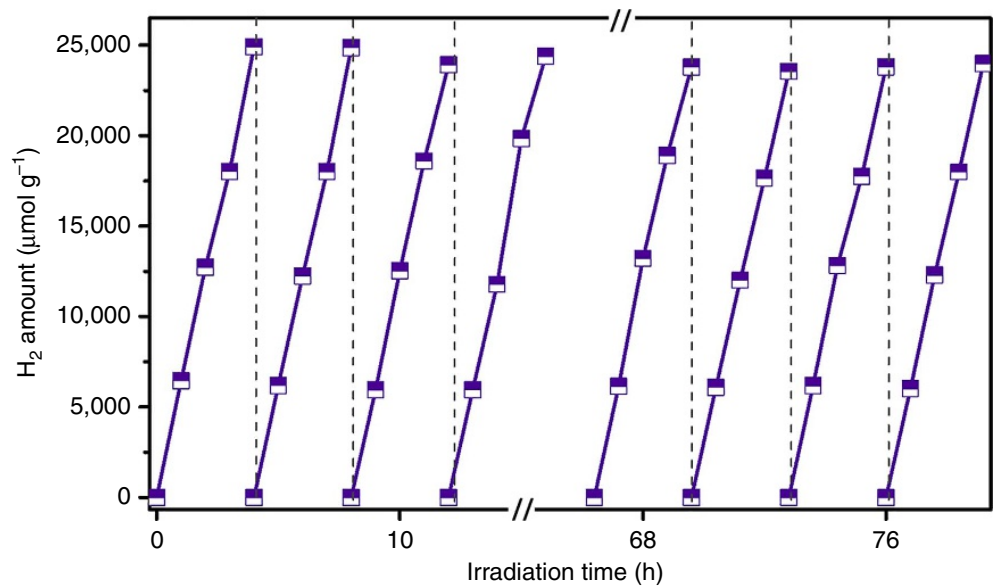

Figure 5 | Photocatalytic $\mathbf{H}_{\mathbf{2}}$ production performance. (a,b) Photocatalytic $\mathrm{H}_{2}$ evolution over $Z$ nln $\mathrm{n}_{2} \mathrm{~S}_{4}$ nanoflowers, bulk $Z$ nln $\mathrm{S}_{4}$ and single-unit-cell $\mathrm{Znln}_{2} \mathrm{~S}_{4}$ layers (a), and $\mathrm{Znln}_{2} \mathrm{~S}_{4} / \mathrm{MoSe}_{2}$ composites with different weight ratios of $\mathrm{MoSe}_{2}$ (b). (c,d) Comparison of photocatalytic $\mathrm{H}_{2}$ evolution activities over $\mathrm{ZnIn}_{2} \mathrm{~S}_{4} / 1 \% \mathrm{Pt}, \mathrm{ZnIn}_{2} \mathrm{~S}_{4} / 1 \% \mathrm{MoS}_{2}$ and $\mathrm{ZnIn}_{2} \mathrm{~S}_{4} / 1 \% \mathrm{MoSe}_{2}$. (e) Recycling photoactivity test of $\mathrm{Znln} \mathrm{S}_{4} / 1 \% \mathrm{MoSe}_{2}$. Note that the error bars represent the photoactivity s.d. values calculated from triplicate experiments.

Besides, photocatalytic $\mathrm{H}_{2}$ activities of ultrathin $\mathrm{CdIn}_{2} \mathrm{~S}_{4}$ and $\mathrm{In}_{2} \mathrm{~S}_{3}$ layers also exceed that of their bulk counterparts (Supplementary Fig. 42). Self-assembly construction of 2D hetero-layer hybrids, that is, $\mathrm{CdIn}_{2} \mathrm{~S}_{4} / \mathrm{MoSe}_{2}$ and $\mathrm{In}_{2} \mathrm{~S}_{3} / \mathrm{MoSe}_{2}$, also verifies the enhanced photoactivity and stability. Essentially, the coupling of ultrathin $\mathrm{CdIn}_{2} \mathrm{~S}_{4}$ and $\operatorname{In}_{2} \mathrm{~S}_{3}$ with a few layers of $\mathrm{MoSe}_{2}$ co-catalyst has enhanced the $\mathrm{H}_{2}$ evolution activity (Supplementary Fig. 43). The rates of $\mathrm{H}_{2}$ evolved over the optimal $\mathrm{CdIn}_{2} \mathrm{~S}_{4} / 1 \% \mathrm{MoSe}_{2}$ and $\mathrm{In}_{2} \mathrm{~S}_{3} / 1 \% \mathrm{MoSe}_{2}$ are $\sim 9$ - and 10 -fold of bare $\mathrm{CdIn}_{2} \mathrm{~S}_{4}$ and $\mathrm{In}_{2} \mathrm{~S}_{3}$ nanosheets, respectively. In addition, the photoactivities of the resulted metal sulfide/ $\mathrm{MoSe}_{2}$ $\left(\mathrm{CdIn}_{2} \mathrm{~S}_{4} / 1 \% \mathrm{MoSe}_{2}\right.$ and $\left.\mathrm{In}_{2} \mathrm{~S}_{3} / 1 \% \mathrm{MoSe}_{2}\right)$ are also much higher than those of the reference photocatalysts, that is, metal sulfide/Pt and metal sulfide/ $\mathrm{MoS}_{2}$ (Supplementary Fig. 44). Furthermore, the cycling tests of the optimal $\mathrm{CdIn}_{2} \mathrm{~S}_{4} / 1 \% \mathrm{MoSe}_{2}$ and $\mathrm{In}_{2} \mathrm{~S}_{3} / 1 \% \mathrm{MoSe}_{2}$ show negligible photoactivity degradation after 20 consecutive cycles with accumulatively $80 \mathrm{~h}$ under visible light irradiation (Supplementary Fig. 45).

\section{Discussion}

Although prior literature has already reported exfoliated 2D layered metal chalcogenides commonly induced by intercalator osmotic swelling, self-surface charge exfoliation into single-unitcell thick layer structure in pure water is unprecedented. Successive artificially coupled hetero-layered structure with ultrathin and intimate interface characteristics, guided by unconstraint electrostatic coordination of a dissimilar metal chalcogenide, is demonstrated in this work. In contrast, thin-film epitaxial growth is strongly influenced by the surface of the substrate and degree of lattice matching. This limits the production yield and imposes a high cost of 2D hetero-layer metal chalcogenide for practical implementation. To test the hypothesis of the satisfactorily high quality of the as-prepared $2 \mathrm{D}$ hetero-layer, surface and interfacial-dominated photocatalysis is used as an ideal testbed for reliability verification. Diverse 2D ultrathin metal sulfide/ $\mathrm{MoSe}_{2}$ hetero-layered materials reveal outstanding visible-light photoreactivity and efficient charge transfer exceeding that of metal sulfide/Pt and metal sulfide/ $\mathrm{MoS}_{2}$ reference photocatalysts. More remarkably, the ultrathin hetero-layer structures demonstrate highly stable contact interface promising for long-term cycling and storage purposes.

In summary, we have developed a scalable method to artificially coordinate ultrathin metal chalcogenide hetero-layer structure via a combination of pristine self-surface charge exfoliation and electrostatic coupling of dissimilar layers. This generic approach to the preparation of $2 \mathrm{D}$ hetero-layered hybrids is attractive as it allows selection of individual constituent materials and thickness control, opening up the possibility of 'design-and-build' 2D layered heterojunction for large-scale theoretical exploration and practical applications. 


\section{Methods}

Materials. Zinc acetate dehydrate $\left(\mathrm{Zn}\left(\mathrm{CH}_{3} \mathrm{COO}\right)_{2} \cdot 2 \mathrm{H}_{2} \mathrm{O}, \geq 98 \%\right)$, sodium molybdate dehydrate $\left(\mathrm{Na}_{2} \mathrm{MoO}_{4} \cdot 2 \mathrm{H}_{2} \mathrm{O}, \geq 99 \%\right)$ and hydrazine hydrate $\left(\mathrm{N}_{2} \mathrm{H}_{4} \cdot \mathrm{H}_{2} \mathrm{O}, 99.99 \%\right)$ solution were obtained from Sigma-Aldrich. Indium chloride $\left(\mathrm{InCl}_{3}, 99.995 \%\right), \mathrm{L}(+)$ lactic acid $(90 \%)$ and selenium $(99.5+\%)$ were obtained from ACROS Organics. Thioacetamide $\left(\mathrm{C}_{2} \mathrm{H}_{5} \mathrm{NS},>98 \%\right)$ was obtained from TCI. All of the reagents were used as received without further purification. The DI water used in the catalyst preparation was from local sources.

Synthesis of single-unit-cell $\mathbf{Z n I n}_{2} \mathbf{S}_{\mathbf{4}}$ layers. Single-unit-cell $\mathrm{ZnIn}_{2} \mathrm{~S}_{4}$ layers were fabricated by a facile low-temperature refluxing method followed by a moderate exfoliation. In detail, $1.5 \mathrm{mmol}$ of $\mathrm{Zn}\left(\mathrm{CH}_{3} \mathrm{COO}\right)_{2} \cdot 2 \mathrm{H}_{2} \mathrm{O}$ and $3 \mathrm{mmol}$ of $\mathrm{InCl}_{3}$ were added into $250 \mathrm{ml}$ DI water and stirred for $30 \mathrm{~min}$. Subsequently, an excess amount of thioacetamide (TAA, $8 \mathrm{mmol}$ ) was added into the above solution and stirred for another $30 \mathrm{~min}$. The solution was then heated to $95^{\circ} \mathrm{C}$ and maintained at that temperature for $5 \mathrm{~h}$ under vigorous stirring. The resulted precipitation was collected by centrifugation, rinsed with water for 2 times and re-dispersed into $200 \mathrm{ml}$ DI water. The dispersion was sonicated continuously for $30 \mathrm{~min}$ and then centrifuged at 6,000 r.p.m. for $5 \mathrm{~min}$ to remove aggregates. After that, the colloidal single-unit-cell $\mathrm{ZnIn}_{2} \mathrm{~S}_{4}$ layers were obtained. For each set of experimental synthesis, $\sim 0.6 \mathrm{~g}$ of $\mathrm{ZnIn}_{2} \mathrm{~S}_{4}$ can be obtained. The comparative sample of $\mathrm{ZnIn}_{2} \mathrm{~S}_{4}$-S was synthesized via the same procedure except that a stoichiometric amount of TAA $(6 \mathrm{mmol})$ was added during the synthesis process.

Synthesis of hetero-layered $\mathbf{Z n I n}_{\mathbf{2}} \mathbf{S}_{\mathbf{4}} / \mathbf{M o S e}_{\mathbf{2}}$ structure. $\mathrm{MoSe}_{2}$ was synthesized via a one-step hydrothermal method ${ }^{44}$. The surface modification of $\mathrm{MoSe}_{2}$ was carried out as follows: $50 \mathrm{mg} \mathrm{MoSe}$ was dispersed in $100 \mathrm{ml}$ ethanol and sonicated continuously for $1 \mathrm{~h}$. Then, $0.25 \mathrm{ml}$ of APTES was added into the above $\mathrm{MoSe}_{2}$ dispersion. The mixture was heated at $60^{\circ} \mathrm{C}$ for $4 \mathrm{~h}$ under mild stirring. The resulted product was rinsed with ethanol for 3 times and redispersed in $100 \mathrm{ml}$ DI water with the aid of ultrasonication for $1 \mathrm{~h}$. After that, the dispersion was centrifuged at 6,000 r.p.m. for $5 \mathrm{~min}$ to remove aggregates. Then, the colloid APTES-modified $\mathrm{MoSe}_{2}$ with positive surface charge was obtained. The $2 \mathrm{D}$ heterolayer composite can be built up by dipping of $\mathrm{MoSe}_{2}$ nanosheet suspension into colloidal $\mathrm{ZnIn}_{2} \mathrm{~S}_{4}$ of constraint supply or controlled amount of dilute solution. In brief, the APTES-modified $\mathrm{MoSe}_{2}$ was added dropwise into the negatively charged $\mathrm{ZnIn}_{2} \mathrm{~S}_{4}$ colloid slowly. Driving by the strong electrostatic attractive interaction, the ultrathin $\mathrm{ZnIn}_{2} \mathrm{~S}_{4}$ nanosheets can self-assemble with the $\mathrm{MoSe}_{2}$ layers, forming intimately integrated $\mathrm{ZnIn}_{2} \mathrm{~S}_{4} / \mathrm{MoSe}_{2}$ hetero-layer structure.

Synthesis of ultrathin $\mathbf{C d I n _ { 2 }} \mathbf{S}_{\mathbf{4}}$ and $\operatorname{In}_{\mathbf{2}} \mathbf{S}_{\mathbf{3}}$ layers. Ultrathin $\mathrm{CdI} \mathrm{In}_{2} \mathrm{~S}_{4}$ layers were fabricated via the similar facile low-temperature refluxing method followed by moderate exfoliation. In detail, $1.5 \mathrm{mmol}$ of $\mathrm{Cd}\left(\mathrm{CH}_{3} \mathrm{COO}\right)_{2} \cdot 2 \mathrm{H}_{2} \mathrm{O}$ and $3 \mathrm{mmol}$ of $\mathrm{InCl}_{3}$ were added into $250 \mathrm{ml}$ DI water and stirred for $30 \mathrm{~min}$. Subsequently, an excess amount of thioacetamide (TAA, $8 \mathrm{mmol}$ ) was added into the above solution and stirred for another $30 \mathrm{~min}$. The solution was then heated to $100{ }^{\circ} \mathrm{C}$ and maintained at that temperature for $12 \mathrm{~h}$ under vigorous stirring. The resulted precipitation was collected by centrifugation, rinsed with water for 2 times and redispersed into $200 \mathrm{ml}$ DI water. The dispersion was sonicated continuously for $30 \mathrm{~min}$ and then centrifuged at 6,000 r.p.m. for $5 \mathrm{~min}$ to remove aggregates. After that, the colloidal ultrathin $\mathrm{ZnIn}_{2} \mathrm{~S}_{4}$ layers were obtained. Ultrathin $\operatorname{In}_{2} \mathrm{~S}_{3}$ layers were synthesized via the same method as that of synthesizing $\mathrm{ZnIn}_{2} \mathrm{~S}_{4}$ layers without the addition of $\mathrm{Zn}\left(\mathrm{CH}_{3} \mathrm{COO}\right)_{2} \cdot 2 \mathrm{H}_{2} \mathrm{O}$ precursor.

Synthesis of hetero-layered $\mathbf{C d I n}_{\mathbf{2}} \mathrm{S}_{\mathbf{4}} / \mathbf{M o S e}_{\mathbf{2}}$ and $\operatorname{In}_{\mathbf{2}} \mathrm{S}_{\mathbf{3}} / \mathbf{M o S e}_{\mathbf{2}}$. The heterolayered $\mathrm{CdIn}_{2} \mathrm{~S}_{4} / \mathrm{MoSe}_{2}$ and $\mathrm{In}_{2} \mathrm{~S}_{3} / \mathrm{MoSe}_{2}$ were synthesized following the same procedure as that of preparing of $\mathrm{ZnIn}_{2} \mathrm{~S}_{4} / \mathrm{MoSe}_{2}$.

Characterization. The XRD patterns of the samples were collected on a Philips $\mathrm{X}$-ray diffractometer with $\mathrm{Cu} \mathrm{K} \alpha$ radiation $(\lambda=1.541 \AA$ ). UV-vis absorption spectra were recorded on a Shimadzu UV-3600 UV-vis spectrophotometer. XPS measurement was performed on a Thermo Scientific ESCA Lab 250 spectrometer that consists of a monochromatic $\mathrm{Al} \mathrm{K} \alpha$ as the $\mathrm{X}$-ray source, a hemispherical analyser and sample stage with multiaxial adjustability to obtain the surface composition of the samples. All of the binding energies were calibrated by the $\mathrm{C} 1 \mathrm{~s}$ peak at $284.6 \mathrm{eV}$. Zeta-potential $(\xi)$ measurements of the samples were determined by dynamic light scattering analysis (Zeta sizer 3000HSA) at a room temperature of $25^{\circ} \mathrm{C}$. SEM images were taken on a JEOL JSM-7001F field emission scanning electron microscope. HRTEM images, EDX and elemental mapping images were obtained on a JEOL JEM-2100 electron microscope. The steady-state PL spectra were recorded on a Shimazu RF-5301PC under the excitation of $400 \mathrm{~nm}$. Tapping-mode AFM measurement was performed on a commercial SPM instrument (MPF-3D, Asylum Research, CA, USA).

Time-resolved photoluminescence measurement was performed under excitation of $400 \mathrm{~nm}$ fs pulses. The excitation source is a mode-locked Ti:sapphire laser (Chameleon Ultra II, Coherent) working with repetition rate of $80 \mathrm{MHz}$ and pulse duration of $140 \mathrm{fs}$. The second harmonic generation of $700 \mathrm{~nm}$ output from the laser was employed to excite the samples. The photoluminescence of the samples was collected and detected by a photon-counting photomultiplier (PMA, Picoquant). The emission centred at $495 \mathrm{~nm}$ was selected by a monochrometer (SpectroPro 2300i, Princeton Instrument). The PL decay dynamics were achieved by a time-correlated single photon counting module (TCSPC Picoharp 300, Picoquant).

Photoelectrochemical measurements were performed in a conventional three-electrode quartz cell. A Pt plate was used as counter electrode, and $\mathrm{Ag} / \mathrm{AgCl}$ electrode/saturated calomel electrode were used as reference electrode, whereas the working electrode was prepared on FTO conductor glass. The sample powder $(3 \mathrm{mg})$ was ultrasonicated in $0.5 \mathrm{ml}$ of $N, N$-dimethylformamide (supplied by Sigma-Aldrich) to disperse it evenly to get a slurry. The slurry was spread onto FTO glass with the area of $1 \mathrm{~cm}^{2}$. After air drying, the working electrode was further dried at $90^{\circ} \mathrm{C}$ for $2 \mathrm{~h}$ to improve adhesion. The electrolyte was $0.2 \mathrm{M}$ aqueous $\mathrm{Na}_{2} \mathrm{SO}_{4}$ solution $(\mathrm{pH}=6.8$ ). Linear sweep voltammetry curves were performed in a mixed solution of $10 \%(\mathrm{v} / \mathrm{v})$ lactic acid and $0.2 \mathrm{M}$ aqueous $\mathrm{Na}_{2} \mathrm{SO}_{4}$ solution.

Photocatalytic $\mathbf{H}_{2}$ evolution measurements. With the aid of ultrasonication, $5 \mathrm{mg}$ of photocatalyst, $9 \mathrm{ml} \mathrm{DI}$ water and $1 \mathrm{ml}$ lactic acid were mixed in a $25 \mathrm{ml}$ quartz cylindrical reaction cell to form a homogeneous suspension. Then, the reactor was purged with argon gas for $10 \mathrm{~min}$ before illumination with a $300 \mathrm{~W}$ xenon arc lamp $(\lambda>400 \mathrm{~nm})$. The evolved $\mathrm{H}_{2}$ was analysed using an online gas chromatograph (GC-2014AT, Shimadzu Co., Japan) equipped with a thermal conductivity detector.

The recycling test of catalytic $\mathrm{H}_{2}$ evolution over the as-prepared photocatalyst was performed as follows. After the reaction of the first run under visible light irradiation, the suspension was purged with argon gas for $10 \mathrm{~min}$. The process was carried out for four more cycles. After every five cycles, the photocatalyst was centrifuged and mixed with fresh $9 \mathrm{ml}$ DI water and $1 \mathrm{ml}$ lactic acid for continuous test.

Data availability. The data that support the findings of this study are available from the corresponding author on request.

\section{References}

1. Du, Y. et al. A general method for the large-scale synthesis of uniform ultrathin metal sulphide nanocrystals. Nat. Commun. 3, 1177 (2012).

2. Geim, A. K. \& Novoselov, K. S. The rise of graphene. Nat. Mater. 6, 183-191 (2007)

3. Butler, S. Z. et al. Progress, challenges, and opportunities in two-dimensional materials beyond graphene. ACS Nano 7, 2898-2926 (2013).

4. Huang, J. et al. Oxyhydroxide nanosheets with highly efficient electron-hole pair separation for hydrogen evolution. Angew. Chem. Int. Ed. 55, 2137-2141 (2016)

5. Sun, Y. et al. Fabrication of flexible and freestanding zinc chalcogenide single layers. Nat. Commun. 3, 1057 (2012).

6. Tan, C. \& Zhang, H. Wet-chemical synthesis and applications of non-layer structured two-dimensional nanomaterials. Nat. Commun. 6, 7873 (2015).

7. Liang, L. et al. Single unit cell bismuth tungstate layers realizing robust solar $\mathrm{CO}_{2}$ reduction to methanol. Angew. Chem. 127, 14177-14180 (2015).

8. Chhowalla, M. et al. The chemistry of two-dimensional layered transition metal dichalcogenide nanosheets. Nat. Chem. 5, 263-275 (2013).

9. Zhang, N., Yang, M.-Q., Liu, S., Sun, Y. \& Xu, Y.-J. Waltzing with the versatile platform of graphene to synthesize composite photocatalysts. Chem. Rev. 115, 10307-10377 (2015).

10. Duan, X., Wang, C., Pan, A., Yu, R. \& Duan, X. Two-dimensional transition metal dichalcogenides as atomically thin semiconductors: opportunities and challenges. Chem. Soc. Rev. 44, 8859-8876 (2015).

11. Ma, R. \& Sasaki, T. Two-dimensional oxide and hydroxide nanosheets: controllable high-quality exfoliation, molecular assembly and exploration of functionality. Acc. Chem. Res. 48, 136-143 (2015).

12. Li, B.-W. et al. Engineered interfaces of artificial perovskite oxide superlattices via nanosheet deposition process. ACS Nano 4, 6673-6680 (2010).

13. Ma, W. et al. A superlattice of alternately stacked Ni-Fe hydroxide nanosheets and graphene for efficient splitting of water. ACS Nano 9, 1977-1984 (2015).

14. Onoda, M., Liu, Z., Ebina, Y., Takada, K. \& Sasaki, T. X-ray diffraction study on restacked flocculates from binary colloidal nanosheet systems $\mathrm{Ti}_{0.91} \mathrm{O}_{2}-\mathrm{MnO}_{2}$, $\mathrm{Ca}_{2} \mathrm{Nb}_{3} \mathrm{O}_{10}-\mathrm{Ti}_{0.91} \mathrm{O}_{2}$ and $\mathrm{Ca}_{2} \mathrm{Nb}_{3} \mathrm{O}_{10}-\mathrm{MnO}_{2}$. J. Phys. Chem. C 115 , 8555-8566 (2011).

15. Chen, J. et al. One-pot synthesis of CdS nanocrystals hybridized with singlelayer transition-metal dichalcogenide nanosheets for efficient photocatalytic hydrogen evolution. Angew. Chem. Int. Ed. 54, 1210-1214 (2015).

16. Palummo, M., Bernardi, M. \& Grossman, J. C. Exciton radiative lifetimes in two-dimensional transition metal dichalcogenides. Nano Lett. 15, 2794-2800 (2015).

17. Deng, D. et al. Catalysis with two-dimensional materials and their heterostructures. Nat. Nanotech. 11, 218-230 (2016).

18. Tan, C. et al. Liquid-phase epitaxial growth of two-dimensional semiconductor hetero-nanostructures. Angew. Chem. Int. Ed. 54, 1841-1845 (2015). 
19. Navarro-Moratalla, E. et al. Enhanced superconductivity in atomically thin $\mathrm{TaS}_{2}$. Nat. Commun. 7, 11043 (2016).

20. Wang, H., Yuan, H., Hong, S. S., Li, Y. \& Cui, Y. Physical and chemical tuning of two-dimensional transition metal dichalcogenides. Chem. Soc. Rev. 44, 2664-2680 (2015).

21. Zheng, J. et al. High yield exfoliation of two-dimensional chalcogenides using sodium naphthalenide. Nat. Commun. 5, 2995 (2014).

22. Hanlon, D. et al. Liquid exfoliation of solvent-stabilized few-layer black phosphorus for applications beyond electronics. Nat. Commun. 6, 8563 (2015).

23. Smith, R. J. et al. Large-scale exfoliation of inorganic layered compounds in aqueous surfactant solutions. Adv. Mater. 23, 3944-3948 (2011)

24. Wang, M., Ioccozia, J., Sun, L., Lin, C. \& Lin, Z. Inorganic-modified semiconductor $\mathrm{TiO}_{2}$ nanotube arrays for photocatalysis. Energy Environ. Sci. 7, 2182-2202 (2014)

25. Hu, J. et al. A versatile strategy for shish-kebab-like multi-heterostructured chalcogenides and enhanced photocatalytic hydrogen evolution. J. Am. Chem. Soc. 137, 11004-11010 (2015).

26. Wang, M. et al. One-dimensional densely aligned perovskite-decorated semiconductor heterojunctions with enhanced photocatalytic activity. Small 11, 1436-1442 (2015).

27. Wang, M., Ye, M., Iocozzia, J., Lin, C. \& Lin, Z. Plasmon-mediated solar energy conversion via photocatalysis in noble metal/semiconductor composites. Adv. Sci. 3, 1600024 (2016)

28. Zhu, L., Tan, C. F., Gao, M. \& Ho, G. W. Design of a metal oxide-organic framework (MoOF) foam microreactor: solar-induced direct pollutant degradation and hydrogen generation. Adv. Mater. 27, 7713-7719 (2015).

29. Wang, J. \& Ho, G. W. Corrosion-mediated self-assembly (CMSA): direct writing towards sculpturing of $3 \mathrm{D}$ tunable functional nanostructures. Angew. Chem. Int. Ed. 54, 15804-15808 (2015).

30. Gao, M., Connor, P. K. N. \& Ho, G. W. Plasmonic photothermic directed broadband sunlight harnessing for seawater catalysis and desalination. Energy Environ. Sci. 9, 3151-3160 (2016)

31. Yang, M.-Q. \& Xu, Y.-J. Photocatalytic conversion of $\mathrm{CO}_{2}$ over graphene-based composites: current status and future perspective. Nanoscale Horiz. 1, 185-200 (2016).

32. Yang, M.-Q., Han, C. \& Xu, Y.-J. Insight into the effect of highly dispersed $\mathrm{MoS}_{2}$ versus layer-structured $\mathrm{MoS}_{2}$ on the photocorrosion and photoactivity of CdS in graphene-CdS-MoS 2 composites. J. Phys. Chem. C 119, 27234-27246 (2015).

33. Yang, J., Wang, D., Han, H. \& Li, C. Roles of cocatalysts in photocatalysis and photoelectrocatalysis. Acc. Chem. Res. 46, 1900-1909 (2013).

34. Kadlag, K. P., Rao, M. J. \& Nag, A. Ligand-free, colloidal, and luminescent metal sulfide nanocrystals. J. Phys. Chem. Lett. 4, 1676-1681 (2013).

35. Grandhi, G. K., Swathi, K., Narayan, K. S. \& Viswanatha, R. Cu doping in ligand free CdS nanocrystals: conductivity and electronic structure study. J. Phys. Chem. Lett. 5, 2382-2389 (2014).

36. Bavykin, D. V., Savinov, E. N. \& Parmon, V. N. Surface effects on regularities of electron transfer in $\mathrm{CdS}$ and $\mathrm{CdS} / \mathrm{Cu}_{\mathrm{x}} \mathrm{S}$ colloids as studied by photoluminescence quenching. Langmuir 15, 4722-4727 (1999).

37. Xu, B. et al. A $1 \mathrm{D} / 2 \mathrm{D}$ helical $\mathrm{CdS} / \mathrm{ZnIn}_{2} \mathrm{~S}_{4}$ nano-heterostructure. Angew. Chem. Int. Ed. 53, 2339-2343 (2014)

38. Yang, M.-Q., Zhang, N., Pagliaro, M. \& Xu, Y.-J. Artificial photosynthesis over graphene-semiconductor composites. Are we getting better? Chem. Soc. Rev. 43, 8240-8254 (2014).

39. Li, L. et al. Layer-by-layer assembly and spontaneous flocculation of oppositely charged oxide and hydroxide nanosheets into inorganic sandwich layered materials. J. Am. Chem. Soc. 129, 8000-8007 (2007).

40. Cai, X. et al. Tuning the surface charge of $2 \mathrm{D}$ oxide nanosheets and the bulk-scale production of superlatticelike composites. J. Am. Chem. Soc. 137, 2844-2847 (2015).

41. Zhang, N. et al. Near-field dielectric scattering promotes optical absorption by platinum nanoparticles. Nat. Photonics 10, 473-482 (2016).

42. Lee, J. S., You, K. H. \& Park, C. B. Highly photoactive, low bandgap $\mathrm{TiO}_{2}$ nanoparticles wrapped by graphene. Adv. Mater. 24, 1084-1088 (2012).

43. Ong, W.-J., Tan, L.-L., Chai, S.-P., Yong, S.-T. \& Mohamed, A. R. Surface charge modification via protonation of graphitic carbon nitride $\left(g-\mathrm{C}_{3} \mathrm{~N}_{4}\right)$ for electrostatic self-assembly construction of $2 \mathrm{D} / 2 \mathrm{D}$ reduced graphene oxide (rGO)/g- $\mathrm{C}_{3} \mathrm{~N}_{4}$ nanostructures toward enhanced photocatalytic reduction of carbon dioxide to methane. Nano Energy 13, 757-770 (2015).

44. Tang, H., Dou, K., Kaun, C.-C., Kuang, Q. \& Yang, S. MoSe ${ }_{2}$ nanosheets and their graphene hybrids: synthesis, characterization and hydrogen evolution reaction studies. J. Mater. Chem. A 2, 360-364 (2014).

45. Xu, C. et al. Ultrathin S-doped $\mathrm{MoSe}_{2}$ nanosheets for efficient hydrogen evolution. J. Mater. Chem. A 2, 5597-5601 (2014).

46. Mao, S. et al. Perpendicularly oriented $\mathrm{MoSe}_{2} /$ graphene nanosheets as advanced electrocatalysts for hydrogen evolution. Small 11, 414-419 (2015).

47. Guo, J., Shi, Y., Bai, X., Wang, X. \& Ma, T. Atomically thin $\mathrm{MoSe}_{2} /$ graphene and $\mathrm{WSe}_{2}$ /graphene nanosheets for the highly efficient oxygen reduction reaction. J. Mater. Chem. A 3, 24397-24404 (2015).
48. Shaw, J. C. et al. Chemical vapor deposition growth of monolayer $\mathrm{MoSe}_{2}$ nanosheets. Nano Res. 7, 511-517 (2014).

49. Lu, X. et al. Rapid and nondestructive identification of polytypism and stacking sequences in few-layer molybdenum diselenide by Raman spectroscopy. $A d v$. Mater. 27, 4502-4508 (2015).

50. Chen, W., Liu, T.-Y., Huang, T., Liu, X.-H. \& Yang, X.-J. Novel mesoporous P-doped graphitic carbon nitride nanosheets coupled with $\mathrm{ZnIn}_{2} \mathrm{~S}_{4}$ nanosheets as efficient visible light driven heterostructures with remarkably enhanced photo-reduction activity. Nanoscale 8, 3711-3719 (2016).

51. Zhang, Z., Liu, K., Feng, Z., Bao, Y. \& Dong, B. Hierarchical sheet-on-sheet $\mathrm{ZnIn}_{2} \mathrm{~S}_{4} / \mathrm{g}-\mathrm{C}_{3} \mathrm{~N}_{4}$ heterostructure with highly efficient photocatalytic $\mathrm{H}_{2}$ production based on photoinduced interfacial charge transfer. Sci. Rep. 6, 19221 (2016).

52. Yuan, L., Yang, M.-Q. \& Xu, Y.-J. A low-temperature and one-step method for fabricating $\mathrm{ZnIn}_{2} \mathrm{~S}_{4}$-GR nanocomposites with enhanced visible light photoactivity. J. Mater. Chem. A 2, 14401-14412 (2014).

53. Gao, M.-R., Chan, M. K. Y. \& Sun, Y. Edge-terminated molybdenum disulfide with a 9.4-A interlayer spacing for electrochemical hydrogen production. Nat. Commun. 6, 7493 (2015).

54. Zhang, Z. et al. Multichannel-improved charge-carrier dynamics in welldesigned hetero-nanostructural plasmonic photocatalysts toward highly efficient solar-to-fuels conversion. Adv. Mater. 27, 5906-5914 (2015)

55 . $\mathrm{Bi}, \mathrm{W}$. et al. Insight into electrocatalysts as co-catalysts in efficient photocatalytic hydrogen evolution. ACS Cata. 6, 4253-4257 (2016)

56. Zhang, N., Yang, M.-Q., Tang, Z.-R. \& Xu, Y.-J. Toward improving the graphene-semiconductor composite photoactivity via the addition of metal ions as generic interfacial mediator. ACS Nano 8, 623-633 (2014).

57. Han, C., Chen, Z., Zhang, N., Colmenares, J. C. \& Xu, Y.-J. Hierarchically CdS decorated $1 \mathrm{D} \mathrm{ZnO}$ nanorods-2D graphene hybrids: low temperature synthesis and enhanced photocatalytic performance. Adv. Funct. Mater. 25, 221-229 (2015).

58. Xiao, F.-X., Miao, J. \& Liu, B. Layer-by-layer self-assembly of CdS quantum dots/graphene nanosheets hybrid Films for photoelectrochemical and photocatalytic applications. J. Am. Chem. Soc. 136, 1559-1569 (2014).

59. Zheng, D. et al. Unconventional route to hairy plasmonic/semiconductor core/shell nanoparticles with precisely controlled dimensions and their use in solar energy conversion. Chem. Mater. 27, 5271-5278 (2015).

60. Song, Y. et al. Enhanced visible-light photocatalytic hydrogen evolution activity of $\mathrm{Er}^{3+}: \mathrm{Y}_{3} \mathrm{Al}_{5} \mathrm{O}_{12} / \mathrm{PdS}-\mathrm{ZnS}$ by conduction band co-catalysts $\left(\mathrm{MoO}_{2}, \mathrm{MoS}_{2}\right.$ and $\mathrm{MoSe}_{2}$ ). Int. J. Hydrogen Energy 41, 12826-12835 (2016).

\section{Acknowledgements}

This work is supported by Ministry of Education (MOE) R-263-000-B38-112 and R-263-000-B63-112.

\section{Author contributions}

G.W.H. proposed the research direction and supervised the project. M.-Q.Y. designed and preformed the experiments. Y.-J.X. performed XPS measurement and provided helpful suggestions in conducting the study. W.H.L. and K.Y.Z. carried out AFM characterization and analysis. H.Z. and Q.-H.X. performed the PL lifetime measurement and analysis. G.W.H. and M.-Q.Y. wrote and revised the manuscript. All authors participated in discussion and reviewed the manuscript before submission.

\section{Additional information}

Supplementary Information accompanies this paper at http://www.nature.com/ naturecommunications

Competing financial interests: The authors declare no competing financial interests

Reprints and permission information is available online at http://npg.nature.com/ reprintsandpermissions/

How to cite this article: Yang, M.-Q. et al. Self-surface charge exfoliation and electrostatically coordinated 2D hetero-layered hybrids. Nat. Commun. 8, 14224 doi: 10.1038/ncomms14224 (2017).

Publisher's note: Springer Nature remains neutral with regard to jurisdictional claims in published maps and institutional affiliations.

This work is licensed under a Creative Commons Attribution 4.0 International License. The images or other third party material in this article are included in the article's Creative Commons license, unless indicated otherwise in the credit line; if the material is not included under the Creative Commons license, users will need to obtain permission from the license holder to reproduce the material To view a copy of this license, visit http://creativecommons.org/licenses/by/4.0/

C) The Author(s) 2017 